\title{
Augmented Reality Tracking Techniques: A Systematic Literature Review Protocol
}

\author{
Ihsan Rabbi ${ }^{1,2}$, Sehat Ullah ${ }^{1}$, Siffat Ullah Khan ${ }^{1,3}$ \\ ${ }^{I}$ (Department of Computer Science and IT / University of Malakand, Pakistan) \\ ${ }_{2}^{2}$ (Institute of Engineering and Computing Sciences / University of Science and Technology, Bannu, Pakistan) \\ ${ }_{3}^{3}$ (Department of Software Engineering / University of Malakand, Pakistan)
}

\begin{abstract}
Context-Augmented Reality (AR) is a technology through which the view of real world environment is augmented by computer generated elements/objects. Tracking and registration are the key challenges in AR system.

Objective- This research paper aims to perform a Systematic Literature Review (SLR) for the identification of challenges during tracking an object in augmented reality environment and how these challenges can be overcame.

Method-We have used a Systematic Literature Review (SLR) for Augmented Reality Tracking Techniques (ARTT). SLR is based on a structured protocol, and is therefore, different from ordinary literature review. We have developed the SLR protocol and are in the process of its implementation.

Expected Outcome-The expected outcomes of the review will be the identification of a list of challenges and its proposed solutions while tacking objects in augmented reality environment.
\end{abstract}

Keywords - Systematic Literature Review, Augmented Reality, Tracking

\section{INTRODUCTION}

Augmented Reality (AR) is a technology through which the view of real world environment is augmented by computer generated elements/objects. AR is related to a mediated reality, in which a view of reality is modified using a computer system. By contrast, virtual reality replaces the real world with a simulated one [1]. In the Reality-Virtuality Continuum (Figure 1) by Milgram et al (1994), augmented reality lies between the virtual world and real world[2].

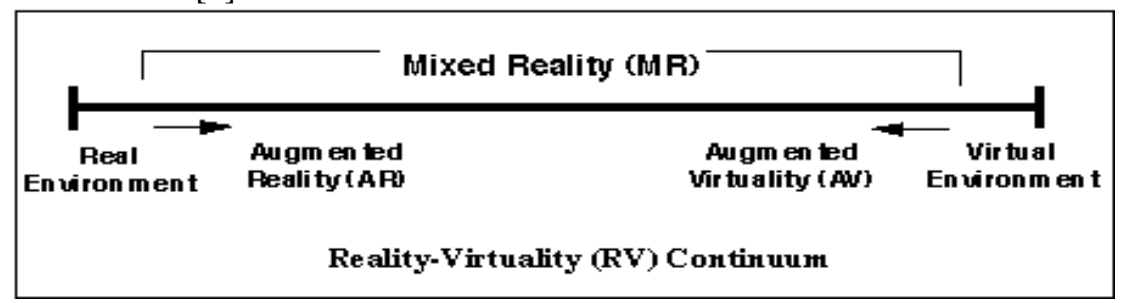

Figure 1. Simplified Representation Of An Reality-Virtuality Continuum [2]

This paper describes the systematic literature review (SLR) [3] protocol which will be used to explore the challenges during tracking objects in the augmented reality system, the proposed solutions presented and their limitations. The goal of our research is to gain in depth understanding of challenges that faced during augmented reality tracking.

Tracking and registration are the most critical issues for AR applications. The proper alignment of virtual objects to the real world environment is called registration [4,5]. Tracking is the main issue for outdoor augmented reality applications [6]. Calibration is another challenge for augmented reality system [7].

Interaction Techniques and User Interfaces are problems for AR system that need to be addressed [8]. Portability of AR system is also a challenge as a wearable system needs to be carried out a whole set of heavy equipment for a long time [9]. The virtual objects and real world environment are required to be of same Illumination [10,11]. Another issue is occlusion, that is a process to determine which surface or its parts are not visible from a certain view-point $[12,13]$

The paper reports our study plan in the form of SLR protocol. This paper presents the design of a first of its kind study in augmented reality tracking techniques. There are two research questions that have motivated the work reported in this paper:

RQ1: What are the challenges, as identified in the literature, for tracking objects in the Augmented Reality system?

RQ2: What are the proposed solutions and its limitations, as identified in the literature, for tracking objects in Augmented Reality system? 


\section{BACKGROUND}

Augmented Reality (AR) is a technology which allows computer-generated virtual objects to exactly overlay real objects in real time. AR is a powerful user interface model that enhances user's perception by augmenting the real world with computer-generated information in real time. AR is a variation of virtual reality. In AR, the user interacts with the real world in a natural way. At the same time the computer is used to generate information related to the real world and the user interacts with computer-generated virtual objects in real time. In contrast, the user of virtual reality completely throws himself into an artificial world [5].

The augmented reality has the following three characteristics [5]:

- Combine real and virtual objects

- Real time interaction

- 3D registration of real and virtual objects

Benford et al (1998) differentiate augmented reality from telepresence and virtual reality (Figure 2) [14].

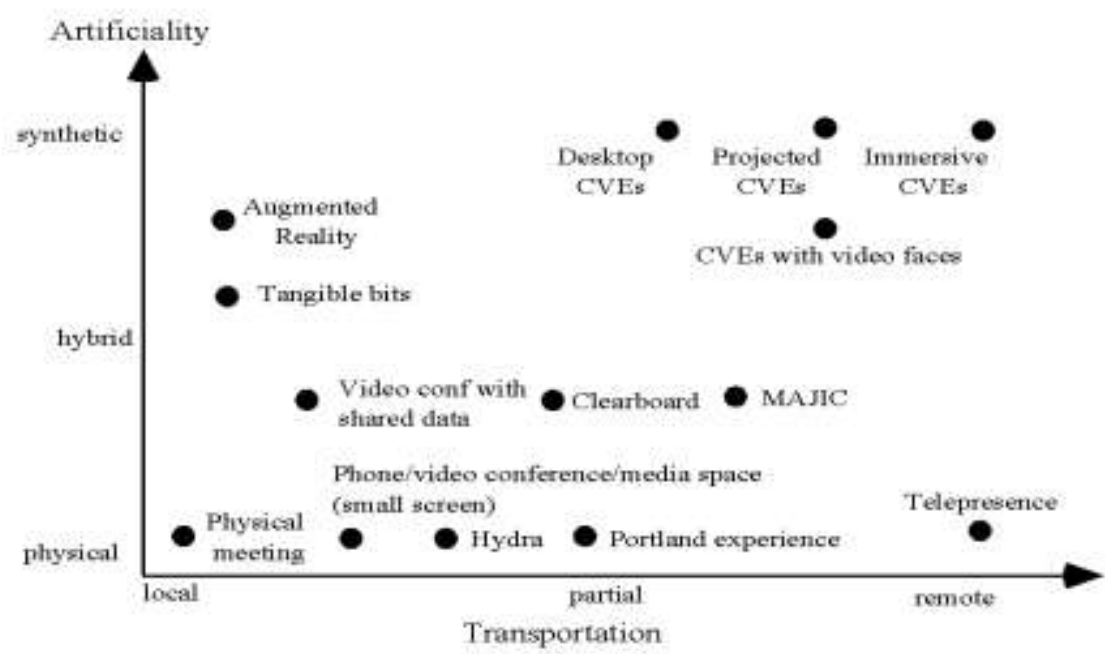

Figure 2. Detailed Classification Of Shared Spaces According To The Dimensions Of Transportation And Artificiality [14]

The combination of virtual and real world in AR is accomplished through Head-Mounted Device (HMD) and trackers. A typical AR system consists of a HMD equipped with one or two cameras. When a user looks around, certain features in the video images captured by the camera are detected to track the camera's position and orientation relative to the objects in the real world. The graphic images generated with this information are rendered on the HMD [5]. A typical wearable computer may be composed of a computer processor and a battery mounted on a belt or backpack, a head mounted display (HMD), wireless communications hardware and an input device such as a touchpad or chording keyboard or voice input capabilities. A wearable computer enables mobility and promises exciting applications with augmented reality. A prominent example is Columbia's "Touring Machine", which assists a user in locating places and allowing a user to query information about items of interest, like campus buildings and library [15].

\subsection{Applications}

In early days augmented reality, the applications of AR were limited only to the fields of medicine, military and industry but now-a-days it is used in almost every field of life. AR is widely used in medical, manufacturing \& repair, annotation \& visualization, robot path planning, entertainment and military aircraft [5]. AR is used almost in every field of life e.g gamming, entertainment, medical, industry, direction finding, identifying object, military, location based communication, consumer design, training, education etc. The detailed discussion about different applications of augmented reality is available in survey papers $[5,16]$.

\subsection{Brief History}

The concept of augmented reality was introduced in 1960's, after the invention of first Head-Mounted Display (HMD) by Ivan Sutherland, which is used as viewing device for augmented reality [17]. The term augmented reality was coined by Tom Caudell and David Mizell in 1990 [18]. KARMA was the first research paper that completely focused on augmented reality system [19]. Rekimoto developed a two dimensional matrix markers that allows camera tracking with six degree of freedom [20]. Mann developed a first GPS-based outdoor system that provides navigational assistance to the visually impaired with spatial audio overlays [21]. Mobile Augmented Reality System (MARS) was developed that registers 3D graphical information in tour guide for buildings and objects [15]. 
Augmented Reality Tracking Techniques: A Systematic Literature Review Protocol

Rasker et al (1998) introduced a spatial augmented reality, in which virtual objects are rendered directly within or on the user's physical space [22]. ARQuake was developed in 2000 which is the first outdoor mobile augmented reality game [23]. AR-PDA is a small wireless augmented reality system [24]. In 2003, a mobile augmented reality system was developed, that guides a user through an unfamiliar building to a certain destination [25]. An indoor augmented reality guidance system was developed in 2003 [26].

Möhring et al (2004) introduced a 3D markers based tracking system using a mobile phone [27]. A hybrid tracking system for outdoor augmented reality in urban environments was developed that enabled accurate, real-time overlays on a handheld device [28]. A parallel real-time tracking and mapping was introduced in 2007 [29]. Wagner et al (2008) developed the first real-time six degree of freedom implementation of natural feature tracking on mobile phones [30]. Morrison et al. (2009) developed MapLens which use magic lens on a paper map to give a mobile augmented reality map [31]. Recently, the field of augmented reality has gained more attention among the researchers.

\section{DESIGNING A SYSTEMATIC LITERATURE REVIEW (SLR) PROTOCOL FOR ARTT}

We have used systematic literature review (SLR) process as a research methodology to review the existing literature in a systematic way. SLR is a new research methodology and is different from ordinary literature review. SLR is comparatively time consuming and difficult to conduct. However, its results are more reliable and less biased. There are three steps in SLR process. The first step is the development of SLR protocol.The designing of SLR protocol for Augmented Reality Tracking Techniques (ARTT) is mainly based on the guidelines as suggested by Kitchenham in 2004 and 2007 [3,32]. A number of protocols were studied for proper guidance [33-36]. Each step of the SLR process is in a pre-defined and structured manner. This includes formation of research question, describing the population, intervention and the outcomes, the development of search terms and search criteria for searching the literature in specified resources, well defined inclusion/exclusion criteria for literature selection, data extraction process and the quality assessment criteria of the selected studies $[3,32]$.

\subsection{Search Strategy}

\subsubsection{Identifying Search Terms}

For the construction of search terms, the following search steps are used.

\begin{tabular}{|l|l|}
\hline Step-A & $\begin{array}{l}\text { Use the Research Questions for the derivation of major terms, } \\
\text { by identifying population, intervention and outcome }\end{array}$ \\
\hline Step-B & $\begin{array}{l}\text { For these major terms, find the alternative spellings and } \\
\text { synonyms }\end{array}$ \\
\hline Step-D & $\begin{array}{l}\text { Use of Boolean Operators for conjunction if the database } \\
\text { allows, in such a way, to use 'OR' operator for the } \\
\text { concatenation of alternative spellings and synonyms whereas } \\
\text { 'AND' for the concatenation of major terms. }\end{array}$ \\
\hline
\end{tabular}


RQ1: The above steps are applied for the construction of search terms for RQ1. The whole procedure is shown as below:

What are the challenges, as identified in the literature, for tracking objects in the augmented reality system?

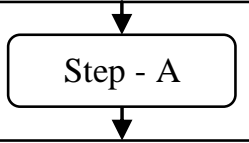

Major Terms: augmented reality system, challenges, tracking objects

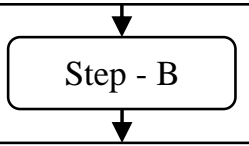

Augmented Reality System: ("augmented reality system" OR AR OR "mixed reality" OR MR OR "supplemented reality" OR "augmented actuality" OR "augmented realism" OR "augmented realness") Challenges: (challenges OR difficulties OR barriers OR hurdles OR threats OR complaints OR hardships OR hardness OR problems OR complications OR obstacles)

Tracking Objects: ("tracking objects" OR "track objects" OR "chasing objects" OR "pursuing objects" OR "tracking items" OR "tracking entities" OR "tracking things" OR "tracking goals" OR "tracking targets" OR "tracking bodies" OR "tracking materials" OR tracker OR "tracking systems")

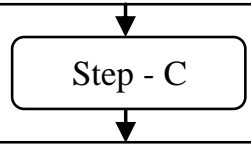

tracking for augmented Reality, challenge for augmented reality is tracking, AR tracker, augmented reality tracking, tracking systems, mixed reality

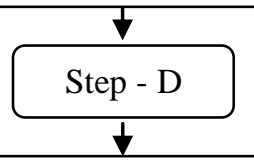

("augmented reality system" OR AR OR "mixed reality" OR MR OR "supplemented reality" OR "augmented actuality" OR "augmented realism" OR "augmented realness") AND (challenges OR difficulties OR barriers OR hurdles OR threats OR complaints OR hardships OR hardness OR problems OR complications OR obstacles) AND ("tracking objects" OR "track objects" OR "chasing objects" OR "pursuing objects" OR "tracking items" OR "tracking entities" OR "tracking things" OR "tracking goals" OR "tracking targets" OR "tracking bodies" OR "tracking materials" OR tracker OR "tracking systems") 
RQ2: Similarly, the above steps are applied for the construction of search terms for RQ2. This procedure is shown as below:

What are the proposed solutions and its limitations, as identified in the literature, for tracking objects in augmented reality system?

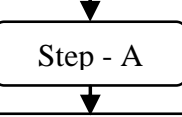

Major Terms: augmented reality system, Proposed Solutions, Limitations, tracking objects

Step - B

Augmented Reality System: ("augmented reality system" OR AR OR "mixed reality" OR MR OR "supplemented reality" OR "augmented actuality" OR "augmented realism" OR "augmented realness")

Proposed Solutions: ("proposed solutions", "nominated solutions", "suggested solutions", present, recommend, design, offer, introduce, "new solutions", "new methods")

Limitations: (limitations, disadvantages, restrictions)

Tracking Objects: ("tracking objects" OR "track objects" OR "chasing objects" OR "pursuing objects" OR "tracking items" OR "tracking entities" OR "tracking things" OR "tracking goals" OR "tracking targets" OR "tracking bodies" OR "tracking materials" OR tracker OR "tracking systems")

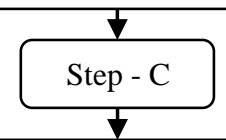

Tracking for Augmented Reality, present a hybrid, Challenge for augmented reality is tracking, AR Tracker, Augmented reality tracking, tracking systems, Mixed reality

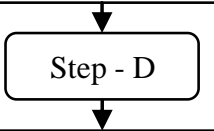

("augmented reality system" OR AR OR "mixed reality" OR MR OR "supplemented reality" OR "augmented actuality" OR "augmented realism" OR "augmented realness") AND ("proposed solutions" OR "nominated solutions" OR "suggested solutions" OR present OR recommend OR design OR offer OR introduce OR "new solutions" OR "new methods" OR limitations OR disadvantages OR restrictions) AND ("tracking objects" OR "track objects" OR "chasing objects" OR "pursuing objects" OR "tracking items" OR "tracking entities" OR "tracking things" OR "tracking goals" OR "tracking targets" OR "tracking bodies" OR "tracking materials" OR tracker OR "tracking systems")

\subsubsection{Resources to be Searched}

IEEEXplore, ACM, Google Scholar, Science Direct, CiteSeer, SpringerLink, Proceedings of International Symposium on Mixed and Augmented Reality (ISMAR).

\subsubsection{Search Constraints and Validation}

We are searching for all published relevant literature to our search terms (strings) using the aforementioned resources. We are searching for all relevant literature and hence do not put any date boundaries. A prior trial search was conducted on ScienceDirect, CiteSeer and IEEExplore digital libraries using a set of major terms ("augmented reality") AND ("tracking objects" OR tracker) AND (challenges OR Solutions OR Limitations).

\subsection{Publication Selection}

Publication selection procedure will be carried out by using publication inclusion criteria, publication exclusion criteria and selection of the primary sources. The main purpose of this publication selection procedure is to choose only those search results which are relevant to our research questions. We will only select those research papers/reports/books that are relating to augmented reality. Others research papers/reports/books not related to augmented reality will be will be ignored.

\subsubsection{Inclusion Criteria}

Inclusion criteria are used to select only that literature (research papers / reports / books) found in the search results obtained. We will only select those research papers related to augmented reality.

The inclusion criteria are listed as below: 
○ Research work that identifies the augmented reality tracking system

- Research work that describe challenges in augmented reality tracking

- Research work that shows different solutions and its limitations for tracking objects in augmented reality system

\subsubsection{Exclusion Criteria}

Exclusion criteria are used to decide which piece of literature (research papers / reports / books) found by the search term will not be selected for review.

The criteria are listed below:

- Research work that is not relevant to our research questions and studies not describing augmented reality tracking

\subsubsection{Selecting Primary Sources}

Primary sources will be initially selected by analyzing the title, keywords and abstracts of searched literature. This review will exclude / ignore those searched literature which have no relevance to the research questions.

The primary sources chosen during this initial selection process will be checked against the above inclusion/ exclusion criteria by reviewing through full text of the research papers.

If any uncertainty occurs regarding the inclusion/ exclusion decision, the case will be sent to the second author. The process will be supervised by the third author.

\subsection{Publication Quality Assessment}

The publication quality assessment is carried out when the final selection of publications is completed. The quality assessment checklist contains the following questions, which will be marked as "Yes" or "No" or "partial" or "NA":

- Is it clearly identify challenges/difficulties during tracking object(s) in the augmented reality environment?

- Is it clearly identify fields/area in the augmented reality tracking environment?

- Is it clear how to solve challenges/difficulties during tracking object(s) in the augmented reality environment?

A second and third reviewer, the authors, will check out a small subset for proper validation.

\subsection{Data Extraction Strategy}

The review will be undertaken by a single researcher (principal author of the paper), who will be responsible for the data extraction. Secondary and tertiary reviewers, the authors, will be approached for guidance in case of an issue regarding the data extraction.

The inter-rater reliability test will be performed after the data extraction process by the primary reviewer. The secondary and tertiary reviewers will select few publications randomly from the list of publication already chosen by the primary reviewer. The reviewers, the authors, will independently extract the data from the randomly selected publication. The results will then be compared with the results produced by the primary reviewer. The reviewers, the authors, will be approached for guidance during the protocol implementation process.

\subsection{Data Synthesis}

Data synthesis will be mainly categorized into two part i.e one for RQ1 and next for RQ2. The first part (for RQ1) will contain background information in one table and the challenges of augmented reality tracking will be placed in other table. The second part of the synthesis (for RQ2) will contain background information in one table whereas the proposed technique used for augmented reality tracking will be kept in another table. SPSS will be used for data analysis.

\section{CONCLUSION AND FUTURE WORK}

Tracking and registration are the most important issues for AR applications that need to be properly addressed. Various studies have shed light on the augmented reality tracking process. However, so far, no systematic literature review process has been conducted for the identification of challenges and limitations for augmented reality tracking process. In this paper we presented our study plan in the form of the SLR protocol. We have developed the SLR protocol and currently we are in the process of its implementation. The findings of this research will provide the challenges and limitations in augmented reality tracking techniques, which will be then addressed to provide a complete solution for tracking objects in augmented reality system.

\section{Acknowledgement}

We are thankful to Software Engineering Research Group at University of Malakand (SERG_UOM) for the review and their valuable comments in validation process of the protocol. SERG_UOM was founded/established by Dr Siffat Ullah Khan in January, 2011. 


\section{References}

[1]. Milgram P, Drascic D, Julius J, et al. Merging Real and Virtual Worlds. Proceedings of IMAGINA '95 (Monte Carlo) 1995:218-230

[2]. Milgram P, Takemura H, Utsumi A, Kishino F. Augmented Reality: A class of displays on the reality-virtuality continuum. In, SPIE Proceedings: Telemanipulator and Telepresence Technologies (Boston, MA); 1994:282-292

[3]. Kitchenham B. Procedures for Performing Systematic Reviews. Joint Technical Report, Keele University TR/SE-0401 and NICTA 0400011T1 2004:33

[4]. Hoff WA, Nguyen K. Computer vision-based registration techniques for augmented reality. Proceedings of Intelligent Robots and Computer Vision XV, SPIE 1996, Boston 1996;2904:538-548

[5]. Azuma RT. A Survey of Augmented Reality. Presence: Teleoperators and Virtual Environments 1997:355-385

[6]. Azuma RT. The Challenge of Making Augmented Reality Work Outdoors. In: Tamura YOaH ed, Mixed Reality: Merging Real and Virtual Worlds. USA: Springer-Verlag; 1999:379-390

[7]. Fuhrmann AL, Splechtna R, Přikryl J. Comprehensive Calibration and Registration Procedures for Augmented Reality. In, In Proceedings Eurographics Workshop on Virtual Environments; 2001

[8]. Zhou F, Duh HB-L, Billinghurst M. Trends in Augmented Reality Tracking, Interaction and Display: A Review of Ten Years of ISMAR. IEEE International Symposium on Mixed and Augmented Reality 2008 2008:193-202

[9]. Schmalstieg D, Fuhrmann A, Hesina G. Bridging Multiple User Interface Dimensions with Augmented Reality. In, In International Symposium of Augmented Reality 2000 (ISAR ‘00); 2000:20-29

[10]. Fournier A. Illumination Problems in Computer Augmented Reality. 1994

[11]. Drettakis G, Robert L, Bougnoux S. Interactive common illumination for computer augmented reality. In 8th Eurographics workshop on Rendering 1997

[12]. Wang X, Dunston PS. Design, Strategies, and Issues towards an Augmented Reality-Based Construction Training Platform. ITcon Vol 12, pg 363-380, http://wwwitconorg/2007/25 2007:363-380

[13]. Fuhrmann A, Hesina G, Faure F, Gervautz M. Occlusion in Collaborative Augmented Environments. Computers and Graphics 1999;23:809-819

[14]. Benford S, Greenhalgh C, Reynard G, Brown C, Koleva B. Understanding and Constructing Shared Spaces with Mixed Reality Boundaries. ACM Trans Computer-Human Interaction 1998:185-223

[15]. Feiner S, MacIntyre B, Höllerer T, Webster A. A touring machine: Prototyping 3D mobile augmented reality systems for exploring the urban environment. Personal and Ubiquitous Computing 1997;1:74-81

[16]. Krevelen DWFV, Poelman R. A Survey of Augmented Reality Technologies, Applications and Limitations. The International Journal of Virtual Reality 2010;9:1-20

[17]. Sutherland IE. A Head Mounted Three Dimensional Display. In, Proceedings of the Fall Joint Computer Conference (AFIPS); 1968:757-764.

[18]. Caudell TP, Mizell DW. Augmented reality: An application of heads-up display technology to manual manufacturing processes. In Proceeding Hawaii International Conference on Systems Sciences, Kauai, HI, USA 1992:659-669

[19]. Feiner S, MacIntyre B, Seligmann D. Knowledge-based augmented reality. Communications of the ACM - Special issue on computer augmented environments: back to the real world 1993;36

[20]. Rekimoto J. Augmented Reality Using the 2D Matrix Code. In Proceedings of the Workshop on Interactive Systems and Software (WISS'96) 1996

[21]. Mann S. Wearable computing: A first step toward personal imaging. Computer 1997;30:25-32

[22]. Raskar R, Welch G, Fuchs H. Spatially Augmented Reality. First International Workshop on Augmented Reality, San Francisco 1998

[23]. Thomas B, Close B, Donoghue J, et al. ARQuake: An Outdoor/Indoor Augmented Reality First Person Application. Proceedings of the Fourth International Symposium on Wearable Computers (ISWC'00) 2000

[24]. Fruend J, Geiger C, Grafe M, Kleinjohann B. The Augmented Reality Personal Digital Assistant. Proceedings of the Second International Symposium on Mixed Reality (ISAR 2001) 2001

[25]. Kalkusch M, Lidy T, Knapp M, et al. Structured Visual Markers for Indoor Pathfinding. Proceedings of the First IEEE International Workshop on ARToolKit (ART02) 2002

[26]. Wagner D, Schmalstieg D. First Steps Towards Handheld Augmented Reality. Proceedings of the 7th IEEE International Symposium on Wearable Computers (ISWC 03) 2003:127-135

[27]. Möhring M, Lessig C, Bimber O. Video See-Through AR on Consumer Cell Phones. Proceedings of the 3th IEEE/ACM international Symposium on Mixed and Augmented Reality (ISMAR 04) 2004:252-253

[28]. Reitmayr G, Drummond T. Going Out: Robust Model-based Tracking for Outdoor Augmented Reality. Proceedings of 5th IEEE and ACM International Symposium on Mixed and Augmented Reality (ISMAR 2006) 2006:109-118

[29]. Klein G, Murray D. Parallel tracking and mapping for small ar workspaces. Proceedings of 6th IEEE and ACM International Symposium on Mixed and Augmented Reality (ISMAR 2007) 2007:225-234

[30]. Wagner D, Reitmayr G, Mulloni A, Drummond T, Schmalstieg D. Pose tracking from natural features on mobile phones. Proceedings of the 7th IEEE/ACM International Symposium on Mixed and Augmented Reality, 2008 (ISMAR 2008) 2008:125-134

[31]. Morrison A, Oulasvirta A, Peltonen P, et al. Like Bees Around the Hive: A Comparative Study of a Mobile Augmented Reality Map. Proceedings of the 27th international conference on Human factors in computing systems (CHI 2009) 2009:1889-1898

[32]. Kitchenham B, Charters S. Guidelines for performing Systematic Literature Reviews in Software Engineering. In, EBSE Technical Report: Software Engineering Group, School of Computer Science and Mathematics, Keele University and Department of Computer Science, University of Durham; 2007

[33]. Khan SU, Niazi M. Systematic Literature Review Protocol for Software Outsourcing Vendors Readiness Model (SOVRM). TR/08 01,School of Computing and Maths, Keele University,UK 2008:28

[34]. Khan SU, Niazi M, Ikram N. Systematic Literature Review Protocol for Software Outsourcing Relationships Trust (SORT). TR/2009-01, School of Computing and Maths, Keele University, UK 2009:40

[35]. Beecham S, Baddoo N, Hall T, Robinson H, Sharp H. Protocol for a Systematic Literature Review of Motivation in Software Engineering. School of Computer Science, University of Hertfordshire, College Lane Campus, Hatfield, Hertfordshire AL10 9AB 2006:87

[36]. Turner M, Charters S. Protocol for a Systematic Literature Review of the Technology Acceptance Model and its Predictive Capabilities. In: Keele University, UK; 2006 